\title{
Analysis of Cardiovascular Risk Factors in Adults with Congenital Heart Disease
}

\author{
Ju Ryoung Moon, RN ${ }^{1}$, Jinyoung Song, MD², June Huh, MD², I-Seok Kang, MD², Seung Woo Park, MD², \\ Sung-A Chang, $\mathrm{MD}^{3}$, Ji-Hyuk Yang, $\mathrm{MD}^{4}$, and Tae-Gook Jun, $\mathrm{MD}^{4}$ \\ ${ }^{1}$ Grown-Up Congenital Heart Clinic, Heart Vascular Stroke Institute, ${ }^{2}$ Department of Pediatrics, Grown-Up Congenital Heart Clinic, Heart Vascular Stroke Institute, \\ ${ }^{3}$ Division of Cardiology, Grown-Up Congenital Heart Clinic, Heart Vascular Stroke Institute, ${ }^{4}$ Department of Thoracic \& Cardiovascular Surgery, Grown-Up Congenital \\ Heart Clinic, Heart Vascular Stroke Institute, Samsung Medical Center, Sungkyunkwan University School of Medicine, Seoul, Korea
}

Background and Objectives: The objective of this study was to analyze cardiovascular risk factors in adults with congenital heart disease (ACHD).

Subjects and Methods: The subjects for this study comprised 135 patients, aged 18 years and above, who visited the ACHD clinic at the Samsung Medical Center and 135 adults with a structurally normal heart who were randomly selected from the Center for Health Promotion during the same period. For the analysis, the ACHD group was further divided into an ACHD group that underwent correction by cardiac surgery and a cyanotic group.

Results: The mean (standard diviation) age (years) of patients in the surgically corrected group was 48.4 (10.9) years, while that of patients in the cyanotic group was 43.1 (9.0) years and that of patients in the control group was 47.1 (10.3) years ( $p=0.042)$. The adjusted odds ratios (ORs) for past smoking, hypertension, diabetes mellitus, hypercholesterolemia, obesity, and metabolic syndrome were significantly higher in the surgically corrected patients than in the controls. However, the ORs for all variables excluding past smoking were significantly lower in the cyanotic group compared with the control group. After adjustment for age, gender, smoking, alcohol use, and exercise, the ORs for metabolic syndrome were $0.46(0.35-0.57, p<0.001)$ and $1.48(1.14-1.92, p=0.003)$ in the cyanotic and surgically corrected groups, respectively.

Conclusion: Cardiovascular risk factors need to be considered in surgically corrected ACHD patients as well as in adults with a structurally normal heart. A further study with a long-term follow-up is needed for developing guidelines for prevention.

\section{(Korean Circ J 2015;45(5):416-423)}

KEY WORDS: Congenital heart disease; Risk factors; Metabolic cardiovascular syndrome.

Received: August 28, 2014

Revision Received: January 21, 2015

Accepted: April 14, 2015

Correspondence: June Huh, MD, Department of Pediatrics, Grown-Up Congenital Heart Clinic, Heart Vascular Stroke Institute, Samsung Medical Center, Sungkyunkwan University School of Medicine, 81 Irwon-ro, Gangnam-gu, Seoul 06351, Korea

Tel: 82-2-3410-3539, Fax: 82-2-3410-0043

E-mail:herzhuh@gmail.com

* The abstract of this article was presented at the 55th Annual Scientific Meeting of the Korean Society of Cardiology.

- The authors have no financial conflicts of interest.

This is an Open Access article distributed under the terms of the Creative Commons Attribution Non-Commercial License (http://creativecommons. org/licenses/ by-nc/3.0) which permits unrestricted non-commercial use, distribution, and reproduction in any medium, provided the original work is properly cited.

\section{Introduction}

Cardiovascular disease (CVD) is the main cause of death and it imposes a burden across the world. ${ }^{1)}$ Developed countries have already reduced the CVD mortality rate considerably through the identification and selection of high-risk groups, and the application of direct interventions, such as education and the prescription of medications. ${ }^{2)}$ However, CVD has been gradually increasing in countries undergoing rapid economic development. ${ }^{2)}$ In Korea, where the ischemic heart disease (IHD) mortality rate is the highest among the CVD mortality rates, the IHD mortality rate has increased sharply from 11.6 persons per 100000 people in 1994 to 28.9 persons per 100000 people in 2012. ${ }^{3)}$ This increase in the IHD mortality rate illustrates the need for an IHD prevention project to actively manage CVD risk factors in Korea. Capitalizing on the 
improved diagnosis and cardiac surgery, 5250 cases of cardiac surgery are performed annually in Korea and a low mortality rate of less than two percent after these surgeries has been recorded. ${ }^{4)}$ As a result, more than $85 \%$ of domestic congenital heart disease (CHD) patients, including cyanotic and complex CHD patients, reach adult age $^{5)}$ and it is estimated that there are already about 150000 to 200000 adults with congenital heart disease (ACHD) patients nationwide. ${ }^{6)}$ However, prevention and management strategies for IHD are still required in these ACHD patients.

Cardiovascular (CV) risk factors include age, hypercholesterolemia, diabetes, hypertension, smoking, drinking, exercise, and metabolic syndrome (MS), and these risk factors are closely related to genetic and environmental predispositions. ${ }^{7)}$ In particular, the identification and management of risk factors attract intense attention because the risk factors for developing IHD after middle age can be mostly prevented or managed by behavioral modifications, such as changes to the lifestyle or dietary habits. ${ }^{7)}$ Furthermore, the identification of risk factors for the specific disease enables tailored care of disease features ${ }_{1}^{8)}$ and may promote more effective prevention or management of conditions such as atherosclerosis and IHD.

Therefore, this research attempted, for the first time in Korea, to provide evidence to support the development of interventions to reduce the $\mathrm{CV}$ risk factors by identifying their levels in ACHD patients and comparing them with those in a control group.

\section{Subjects and Methods}

\section{Study sample}

This study was conducted in 135 ACHD patients and 135 adults with a structurally normal heart who were randomly selected from those who visited the Center for Health Promotion at the hospital during the same period. The ACHD patient group consisted of 135 patients, aged 18 years and above, who visited the outpatient clinic for ACHD at the Samsung Medical Center between October 1, 2010 and April 30, 2011. This group was further divided into two ACHD groups, cyanotic and surgically corrected ACHD groups. The cyanotic ACHD group comprised 45 cyanotic ACHD patients who had cyanosis, regardless of whether or not they underwent cardiac surgery. Cyanosis was defined in patients with a peripheral oxygen saturation of $<93 \%$ in room air, an unrepaired intra- or extracardiac shunt, and severe pulmonary hypertension diagnosed by either echocardiography or cardiac catheterization. The surgically corrected

Table 1. Clinical characteristics of patients in congenital heart disease groups $(\mathrm{N}=135)$

\begin{tabular}{|c|c|c|c|c|}
\hline \multirow{3}{*}{ Variables } & \multirow{3}{*}{ N (\%) } & \multirow{2}{*}{\multicolumn{2}{|c|}{$\begin{array}{c}\text { Cyanotic ACHD }(n=45) \\
\text { Cardiac surgery }\end{array}$}} & \multirow{3}{*}{$\begin{array}{c}\text { Surgically corrected } \\
\text { ACHD }(n=90) \\
\text { Cardiac surgery } \\
\text { Yes }(n=57)\end{array}$} \\
\hline & & & & \\
\hline & & Yes $(n=17)$ & No $(n=28)$ & \\
\hline \multicolumn{5}{|l|}{ Type of CHD } \\
\hline Atrial septal defect & $43(33.1)$ & 3 & 10 & 30 \\
\hline Tetralogy of fallot & $22(16.3)$ & & & 22 \\
\hline Ventricular septal defect & $17(12.6)$ & 2 & 5 & 17 \\
\hline Double outlet right ventricle & $7(5.1)$ & 3 & & 4 \\
\hline Patent ductus arteriosus & $7(5.1)$ & & 7 & \\
\hline Ebstein anomaly & $6(4.3)$ & & & 6 \\
\hline Partial atrioventricular septal defect & $6(4.3)$ & 1 & & 5 \\
\hline L-transposition of the great arteries & $6(4.3)$ & 2 & 1 & 3 \\
\hline Single ventricle & $5(3.7)$ & 2 & & 3 \\
\hline Pulmonary atresia with ventricular septal defect, MAPCA & $4(2.8)$ & 3 & & 1 \\
\hline Complete atrioventricular septal defect & $3(2.1)$ & & 3 & \\
\hline Tricuspid atresia & $3(2.1)$ & & & 3 \\
\hline Pulmonary stenosis & $3(2.1)$ & & & 3 \\
\hline Truncus arteriosus & $2(1.4)$ & & 2 & \\
\hline D-transposition of the great arteries & $1(0.7)$ & 1 & & \\
\hline Duration after cardiac surgery (years) ${ }^{*}$ & & $28.4(14.5)$ & & $31.9(17.2)$ \\
\hline Age at surgery (years) * & & $15.0(15.2)$ & & $18.21(14.6)$ \\
\hline
\end{tabular}

Data are presented as mean (standard deviation). ACHD: adults with congenital heart disease, CHD: congenital heart disease, MAPCA: major aortopulmonary collateral artery 
ACHD group comprised 90 ACHD patients who did not have cyanosis after undergoing total correction via cardiac surgery, regardless of the original CHD. The distribution of original CHD types in each group is shown in Table 1. The proportion of patients who underwent surgery in the cyanotic ACHD group was 37.7\%. The mean \{standard diviation (SD)\} duration after cardiac surgery was 28.4 (14.5) years in the cyanotic ACHD group and 31.9 (17.2) years in the surgically corrected ACHD group. There was no significant difference in the duration after surgery between the two groups (Table 1). The control group was selected by one-to-one pairing based on age, gender, and body mass index (BMI) from patients who visited the Center for Health Promotion at the Samsung Medical Center during the same period. Study data were obtained by reviewing electronic medical records. Informed consent was obtained from the subjects, and the study protocol was approved by the Institutional Review Board of Samsung Medical Center.

\section{Cardiovascular risk factors}

The variables surveyed as CV risk factors included age, gender, weight, height, BMI, systolic blood pressure (SBP), diastolic blood pressure (DBP), total cholesterol (TC), triglycerides (TG), high-density lipoprotein cholesterol (HDL-C), low-density lipoprotein cholesterol $(\mathrm{LDL}-\mathrm{C})$, fasting blood sugar (FBS), diagnosis of hypertension, and diagnosis of diabetes. Variables related to healthy behavior included smoking experience (never, past, current smoker), alcohol use (normal, hazardous, problematic), and physical activity level.

Blood pressure (BP) was measured three times, and the average of the second and third BP readings was used in this study. Patients were defined as having hypertension (HT) if they were taking an antihypertensive drug, had been clinically diagnosed with $\mathrm{HT}$, or had either an SBP $\geq 140 \mathrm{mmHg}$ or a DBP $\geq 90 \mathrm{mmHg}$. Patients who met one of the following criteria were defined as having diabetes mellitus (DM): taking an oral hypoglycemic agent, using insulin, a clinical diagnosis of DM, or an FBS level>126 mg/dL. Patients were defined as having hypercholesterolemia if they met one of the following criteria: diagnosis of hypercholesterolemia or a history of taking medication for hypercholesterolemia, TC $>200 \mathrm{mg} / \mathrm{dL}$, or LDL-C > $130 \mathrm{mg} / \mathrm{dL}$. The following BMl categories ${ }^{9)}$ were recognized: normal $\left(<22.9 \mathrm{~kg} / \mathrm{m}^{2}\right)$, overweight $\left(23.0-24.9 \mathrm{~kg} / \mathrm{m}^{2}\right)$, and obese $\left(\geq 25.0 \mathrm{~kg} / \mathrm{m}^{2}\right)$. With respect to smoking, the subjects were categorized into those who had never smoked, past smokers, and current smokers. ${ }^{10)}$ Alcohol use behavior was categorized according to the Alcohol Use Disorders Identification Test (AUDIT) score. AUDIT is a simple tool, developed by the World Health Organization, ${ }^{11)}$ to identify alcohol use behaviors in a primary care setting. The main items on this instrument are recent alcohol use, alcohol dependence symptoms, and alcohol-related problems. The test consists of 10 questions adding up to a total score ranging from 0 to 40 ; that is, each question has a set of possible responses, and each response has a score ranging from 0 to 4 . Scores in the range of $0-7$ represent low-risk drinking, 8-15 represents a medium-level alcohol problem, and scores of $\geq 16$ represent a high-level alcohol problem. ${ }^{11}$ This study categorized alcohol use into normal use (0-7), hazardous use (8-15), and problematic use (16-40). Exercise was assessed on a yes/no basis, where "exercise" meant moderate activity for more than 30 minutes five days per week, intense activity for more than 20 minutes three days per week ${ }_{1}^{12)}$ or walking for more than 30 minutes a day for more than five days per week.

Based on the guidance provided by the National Cholesterol Education Program - Adult Treatment Panel III ${ }^{13-16)}$ and the Western Pacific Region's Asia Pacific Guideline, ${ }_{1}^{9)}$ we defined MS when three or more of the following criteria were satisfied: 1) presence of hypertension, as determined by SBP $\geq 130 \mathrm{mmHg}$ or DBP $\geq 85$ $\mathrm{mmHg}$, or undergoing active antihypertensive drug therapy; 2) FBS $\geq 100 \mathrm{mg} / \mathrm{dL}$ or active use of oral hypoglycemic agents or insulin; 3 ) TG levels $\geq 150 \mathrm{mg} / \mathrm{dL}$; 4) HDL-C $<40 \mathrm{mg} / \mathrm{dL}$ in males, $<50 \mathrm{mg} / \mathrm{dL}$ in females; and 5) $\mathrm{BMI} \geq 25 \mathrm{~kg} / \mathrm{m}^{2}$. 17/18)

\section{Statistical analysis}

Demographic data and CV risk factors were summarized descriptively with continuous variables expressed as mean (SD), and categorical data were presented as percentage frequency. The general characteristics of subjects and $\mathrm{CV}$ risk factors in the surgically corrected ACHD, cyanotic ACHD, and control groups were analyzed by one-way analysis of variance or Kruskal -Wallis test with the Bonferroni method for performing multiple comparisons of continuous variables. The $\chi^{2}$ test or Fisher exact test was used to compare categorical variables. Multiple logistic regression analysis was employed to determine the association between $\mathrm{CV}$ risk factors in the cyanotic $A C H D$, surgically corrected $A C H D$, and control groups after adjustment for age, gender, smoking, drinking, and exercise. Results were regarded as statistically significant when the $p$ value was $<0.05$. All analyses were performed using statistical software (SAS 9.3; SAS Institute Inc., Cary, NC, USA).

\section{Results}

The mean (SD) age of patients in the surgically corrected ACHD group was 48.4 (10.9) years, while the mean age of patients in the cyanotic ACHD group was 43.1 (9.0) years, and that of patients in the control group was 47.1 (10.3) years ( $p=0.042$ ). The proportion of males was $36.7 \%$ in the surgically corrected ACHD group, $57.8 \%$ in the cyanotic ACHD group, and $43.7 \%$ in the control group 
$(p=0.002)$. The proportion of subjects who had never smoked was highest in the cyanotic ACHD group at $77.8 \%$, and the highest proportion of current smokers was $22.3 \%$, in the control group $(p=0.018)$. The proportion of subjects with alcohol use categorized as hazardous was highest in the surgically corrected ACHD group at $43.3 \%$, and the highest percentage of problematic alcohol use was $16.3 \%$ in the control group $(p=0.027)$. The highest proportion of subjects who exercised was found in the control group (47.4\%)

Table 2. General characteristics of patients and cardiovascular risk factors in cyanotic congenital heart disease, surgically corrected congenital heart disease, and control groups ( $\mathrm{N}=270)$

\begin{tabular}{|c|c|c|c|c|}
\hline Variables & $\begin{array}{c}\text { Cyanotic ACHD } \\
(n=45)\end{array}$ & $\begin{array}{l}\text { Surgically corrected ACHD } \\
\qquad(n=90)\end{array}$ & $\begin{array}{l}\text { Control } \\
(n=135)\end{array}$ & $\mathbf{p}^{*}$ \\
\hline Age (years) & $43.1(9.0)^{a b}$ & $48.4(10.9)^{a}$ & $47.1(10.3)^{b}$ & 0.042 \\
\hline \multicolumn{5}{|l|}{ Gender, n (\%) } \\
\hline Male & $26(57.8)^{\mathrm{ac}}$ & $33(36.7)^{\mathrm{ab}}$ & $59(43.7)^{b c}$ & 0.002 \\
\hline \multicolumn{5}{|l|}{ Smoking, n (\%) } \\
\hline Never & $35(77.8)^{\mathrm{ab}}$ & $54(66.1)^{\mathrm{ab}}$ & $85(62.9)^{b c}$ & 0.018 \\
\hline Past & $9(20.0)^{a b}$ & $21(23.3)^{a}$ & $20(14.8)^{b}$ & \\
\hline Current & $1(2.2)^{\mathrm{ac}}$ & $15(16.6)^{\mathrm{ab}}$ & $30(22.3)^{b c}$ & \\
\hline \multicolumn{5}{|l|}{ Alcohol use, $n(\%)$} \\
\hline Normal & $35(77.7)^{\mathrm{ab}}$ & $37(41.1)^{a}$ & $47(34.8)^{b}$ & \\
\hline Hazardous & $6(13.3)^{a c}$ & $39(43.3)^{\mathrm{ab}}$ & $66(48.9)^{b c}$ & 0.027 \\
\hline Problematic & $4(8.0)^{\mathrm{ab}}$ & $14(15.6)^{\mathrm{a}}$ & $22(16.3)^{b}$ & \\
\hline \multicolumn{5}{|l|}{ Exercise, n (\%) } \\
\hline Yes & $10(22.2)^{a c}$ & $33(37.0)^{\mathrm{ab}}$ & $64(47.4)^{b c}$ & $<0.001$ \\
\hline $\mathrm{SBP}(\mathrm{mmHg})$ & $110.7(14.1)$ & $135.4(15.9)$ & $128.3(16.1)$ & 0.178 \\
\hline $\mathrm{DBP}(\mathrm{mmHg})$ & $69.1(10.7)$ & $80.2(12.1)$ & $79.3(11.4)$ & 0.189 \\
\hline \multicolumn{5}{|l|}{ Cholesterol, n (\%) } \\
\hline $\mathrm{TC}(\mathrm{mg} / \mathrm{dL})$ & $170.9(27.0)^{\mathrm{ab}}$ & $192.8(34.6)^{a}$ & $185.1(33.1)^{b}$ & $<0.001$ \\
\hline $\mathrm{TG}(\mathrm{mg} / \mathrm{dL})$ & $106.5(58.5)^{\mathrm{a}}$ & $122.3(57.3)^{b}$ & $111.9(54.3)^{a b}$ & $<0.001$ \\
\hline $\mathrm{LDL}-\mathrm{C}(\mathrm{mg} / \mathrm{dL})$ & $100.6(28.8)^{a}$ & $118.0(31.6)^{\mathrm{ab}}$ & $104.1(22.9)^{b}$ & $<0.001$ \\
\hline $\mathrm{HDL}-\mathrm{C}(\mathrm{mg} / \mathrm{dL})$ & $46.8(13.1)^{a c}$ & $52.9(12.9)^{\mathrm{ab}}$ & $58.8(15.1)^{b c}$ & $<0.001$ \\
\hline $\mathrm{FBS}(\mathrm{mg} / \mathrm{dL})$ & $103.5(21.2)^{\mathrm{ac}}$ & $99.5(13.1)^{\mathrm{ab}}$ & $95.0(16.1)^{b c}$ & $<0.001$ \\
\hline Hypertension, n (\%) & $1(2.2)^{a c}$ & $36(40.3)^{a b}$ & $47(35.2)^{b c}$ & $<0.001$ \\
\hline Diabetes, n (\%) & $1(2.2)^{\mathrm{ac}}$ & $34(38.5)^{a b}$ & $41(30.3)^{b c}$ & $<0.001$ \\
\hline Hypercholesterolemia, n (\%) & $3(6.0)^{\mathrm{ac}}$ & $35(38.8)^{a b}$ & $47(34.8)^{b c}$ & $<0.001$ \\
\hline \multicolumn{5}{|l|}{ BMI, n (\%) } \\
\hline Overweight & $1(2.2)^{\mathrm{ab}}$ & $19(21.1)^{a}$ & $24(17.7)^{b}$ & $<0.001$ \\
\hline Obese & $1(2.2)^{\mathrm{ac}}$ & $28(31.1)^{a b}$ & $32(23.7)^{b c}$ & $<0.001$ \\
\hline Metabolic syndrome, n (\%) & $5(11.1)^{\mathrm{ac}}$ & $33(36.6)^{a b}$ & $33(24.4)^{b c}$ & 0.015 \\
\hline High BP & $3(6.0)^{a b}$ & $39(43.3)^{a}$ & $55(40.7)^{b}$ & $<0.001$ \\
\hline High FBS & $7(10.2)^{a c}$ & $37(41.1)^{\mathrm{ab}}$ & $48(35.5)^{b c}$ & $<0.001$ \\
\hline High TG & $5(10.1)^{a b}$ & $33(36.6)^{a}$ & $48(35.5)^{b}$ & 0.019 \\
\hline Low HDL-C & $6(13.3)^{a c}$ & $29(32.2)^{a b}$ & $26(21.4)^{b c}$ & $<0.001$ \\
\hline Obese & $1(2.2)^{\mathrm{ac}}$ & $28(31.1)^{\mathrm{ab}}$ & $32(23.7)^{b c}$ & $<0.001$ \\
\hline
\end{tabular}

*Analysis of variance (ANOVA), Kruskal-Wallis test, $\chi^{2}$ test or Fisher exact test for surgically corrected CHD vs. cyanotic CHD vs control groups. $p$ was calculated by one-way ANOVA or Kruskal-Wallis test and Bonferroni tests for multiple comparisons of continuous variables $(p<0.05)$. ${ }^{a}, b, c m e a n s$ with the same letters are significantly different by Bonferroni test. ACHD: adults with congenital heart disease, SBP: systolic blood pressure, DBP: diastolic blood pressur, TC: total cholesterol, TG: triglycerides, LDL-C: low-density lipoprotein cholesterol, HDL-C: high-density lipoprotein cholesterol, FBS: fasting blood sugar, BMI: body mass index, BP: blood pressure 
$(p<0.001)$. The cholesterol measurements showed the highest level of $T C, T G$, and LDL-C in the surgically corrected ACHD group; FBS level was highest in the cyanotic ACHD group ( $p<0.001)$. The corrected ACHD group had a higher proportion of subjects with $\mathrm{HT}$, $\mathrm{DM}$, and hypercholesterolemia than the cyanotic and control group

Table 3. Multivariable analysis of risk factors in patients with congenital heart disease

\begin{tabular}{|c|c|c|}
\hline Variables & OR (95\% Cl) & p \\
\hline \multicolumn{3}{|l|}{ Group } \\
\hline Control & 1.0 & \\
\hline Cyanotic ACHD & $0.46(0.35-0.57)$ & $<0.001$ \\
\hline Surgically corrected ACHD & $1.48(1.14-1.92)$ & 0.003 \\
\hline Age & $1.05(1.03-1.05)$ & $<0.001$ \\
\hline \multicolumn{3}{|l|}{ Gender } \\
\hline Female & 1.0 & \\
\hline Male & $2.31(1.90-2.81)$ & $<0.001$ \\
\hline \multicolumn{3}{|l|}{ Smoking } \\
\hline Never & 1.0 & \\
\hline Past & $1.08(0.95-1.22)$ & 0.238 \\
\hline Current & $1.77(1.54-2.02)$ & $<0.001$ \\
\hline \multicolumn{3}{|l|}{ Alcohol use } \\
\hline Normal & 1.0 & \\
\hline Hazardous & $1.19(1.02-1.40)$ & 0.029 \\
\hline Problematic & $2.14(1.95-2.35)$ & $<0.001$ \\
\hline \multicolumn{3}{|l|}{ Exercise } \\
\hline Yes & 1.0 & \\
\hline No & $2.29(1.99-2.59)$ & $<0.001$ \\
\hline
\end{tabular}

(all $\mathrm{p}<0.001$ ). In addition, more number of subjects in the surgically corrected ACHD group were obese than those in the cyanotic ACHD and control groups $(p<0.001)$. MS had the highest distribution in the surgically corrected ACHD group. Among the components of MS, subjects in the surgically corrected ACHD group had higher BP $(p<0.001)$, higher FBS $(p<0.001)$, higher TG $(p=0.019)$, lower HDL-C $(p<0.001)$, and were more obese $(p<0.001)$ than those in the other two groups. The results after Bonferroni correction for multiple comparisons are shown in Table 2.

There was a tendency for an increased risk of MS in the ACHD groups with age, male gender, alcohol consumption, and a lack of exercise. After adjustment for age, gender, smoking, alcohol use, and exercise, the ORs for MS were $0.46\{95 \%$ confidence interval (CI) $0.35-0.57\}$ and 1.48 (95\% Cl 1.14-1.92) in the cyanotic ACHD and surgically corrected ACHD groups, respectively, with an increase in the surgically corrected ACHD group (Table 3). Additionally, after adjustment for risk factors in the surgically corrected ACHD group, age [OR 2.43, 95\% Cl 1.33-4.42)], male gender [OR 2.51,95\% Cl 1.93-4.76), smoking [OR $1.63,95 \% \mathrm{Cl} 1.05-2.52$ in the current smoking group], alcohol consumption [OR 1.08, 95\% Cl 1.06-1.09) in the hazardous use group; OR $1.96,95 \% \mathrm{Cl} 1.21-2.85$ ) in the problematic use group] and no exercise [OR $2.31,95 \% \mathrm{Cl} 1.40-4.55)$ ] were significantly associated with an increased risk of MS (Table 4).

\section{Discussion}

This research was undertaken to provide support for the development of interventions to lower CV risk factor levels by identifying these levels in ACHD patients and comparing them with those in a control group. This is the first time that such a research

Table 4. Multivariable analysis of risk factors in patients with cyanotic congenital heart disease and surgically corrected congenital heart disease

\begin{tabular}{|c|c|c|c|c|}
\hline \multirow{2}{*}{ Variables } & \multicolumn{2}{|c|}{ Cyanotic ACHD $(n=45)$} & \multicolumn{2}{|c|}{ Surgically corrected ACHD $(n=90)$} \\
\hline & OR $(95 \% \mathrm{Cl})$ & $p$ & OR $(95 \% \mathrm{Cl})$ & $p$ \\
\hline Age & $1.77(1.27-4.17)$ & 0.026 & $2.43(1.33-4.42)$ & 0.004 \\
\hline Gender (male) & $0.07(0.01-1.47)$ & 0.086 & $2.51(1.93-4.76)$ & $<0.001$ \\
\hline \multicolumn{5}{|l|}{ Smoking } \\
\hline Never & 1.0 & & 1.0 & \\
\hline Past & $1.21(0.77-3.29)$ & 0.426 & $1.11(0.73-1.71)$ & 0.091 \\
\hline Current & $1.58(0.89-4.05)$ & 0.590 & $1.63(1.05-2.52)$ & 0.031 \\
\hline \multicolumn{5}{|l|}{ Alcohol use } \\
\hline Normal & 1.0 & & 1.0 & \\
\hline Hazardous & $1.09(0.93-4.84)$ & 0.382 & $1.08(1.06-1.09)$ & $<0.001$ \\
\hline Problematic & $1.97(0.14-9.67)$ & 0.397 & $1.96(1.21-2.85)$ & 0.004 \\
\hline Exercise (No) & $1.11(0.73-3.71)$ & 0.194 & $2.31(1.40-4.55)$ & 0.008 \\
\hline
\end{tabular}

ACHD: adults with congenital heart disease, OR: odds ratio, $\mathrm{Cl}$ : confidence interval 
has been undertaken in Korea. According to the results of this study, the surgically corrected ACHD group had higher levels of risk factors as compared with the control group, but the cyanotic ACHD group had lower levels of risk factors than the control group.

Atherosclerotic CVD is the main cause of mortality in the adult population, and the atherosclerotic process, together with various risk factors, is closely related to lipid concentrations. ${ }^{19)}$ Of these risk factors, being overweight and obese have positive correlations with high lipid levels and total cholesterol, and have negative correlations with HDL-C. ${ }^{20-22)}$ In addition to obesity, a lack of physical activity is also associated with the accumulation of fat and metabolic changes. Vasconcelos et al. ${ }^{23)}$ reported that, compared with inactive or sedentary peers, active adults had lower total cholesterol and TG levels. Moreover, Varady et al. ${ }^{24)}$ stated that through lifestyle changes, including diet and exercise, most adults could significantly decrease their lipid levels and atherosclerotic risk. However, because there was a belief that physical activity or exercise may have a negative influence on their cardiac condition, ACHD patients had a tendency to avoid physical activity or exercise. ${ }^{25)}$ This finding is in partial agreement with the results of our study. Patients in the surgically corrected ACHD group, who exercised less than those in the control group, were more obese and had higher lipid levels. In addition, subjects in the surgically corrected ACHD group had increased risk of hypertension, diabetes, hyperlipidemia, and MS than those in the control group. Therefore, active management of obesity and lipid levels through diet and customized exercise prescriptions, taking into account the patient's cardiac condition, may reduce the prevalence of hypertension, diabetes, and hyperlipidemia.

Unlike the patients in the surgically corrected ACHD group, those in the cyanotic ACHD group in the present study had a significantly lower risk of high lipid levels, including LDL-C, hyperlipidemia, diabetes, hypertension, and metabolic disorder compared to those in the other two groups. This result is in agreement with the finding of the study by Martinez-Quintana et al. ${ }^{20)}$ which suggested that patients with cyanosis had significantly lower levels of TC and LDL-C compared with patients without cyanosis. Hypocholesterolemia can be explained by the genetic determinants of cyanosis, hypoxemia, erythrocytosis and, related factors. In addition, the lower prevalence of coronary atherosclerosis in the cyanotic ACHD group might be explained by hypocholesterolemia, along with the upregulation of nitric oxide, hyperbilirubinemia, and a lower platelet count. ${ }^{26)}$

There are still some controversies about glucose levels. Hait et al. ${ }^{27)}$ reported that glucose levels could be high in patients with a left-to-right shunt lesion such as ASD, VSD, and atrioventricular septal defect as well as even in cyanotic patients including those with Eisenmenger syndrome due to excessive clearance of insulin by the lungs. However, Lundell et al. ${ }^{28)}$ suggested that cyanotic patients can also have lower glucose levels when fasting because of chronic increases in circulating catecholamines and poor nutrition. In our study, cyanotic patients including patients with Eisenmenger syndrome showed significantly higher glucose levels than those in the other two groups. These cyanotic patients could have unstable glucose levels; hence, they require continuous monitoring and nutritional management.

This study showed that the risk of MS in patients of the surgically corrected ACHD group was 1.48 times higher than that in patients of the control group, and that the risk factors included age, gender, lack of exercise, alcohol consumption, and smoking. These factors were the already known risk factors for an increase in MS and they were controllable factors. ${ }^{7)}$ Therefore, during follow-up in the ACHD outpatient clinic, identifying the patient's risk factors, and developing as well as applying tailored intervention which allows self-management of the risk factors may contribute to the prevention of MS in ACHD patients. In a manner similar to that in which hypertension can persist after total correction of coarctation of the aorta, adverse sequelae of a CV event can persist after CHD treatment. For these reasons, patients with $\mathrm{CHD}$ need proactive evaluation of $\mathrm{CV}$ risk factors and more frequent follow-ups. ${ }^{29)}$

Our study has a number of limitations. First, there were significant differences in the age and gender between the participant groups. To minimize the effect of age and gender, an adjusted analysis was conducted. Second, the surgically corrected ACHD group included patients who underwent total correction, irrespective of whether the original CHD morphological classification diagnosis was acyanotic or cyanotic CHD. According to Fyfe et al. ${ }^{26)}$ there was a significant difference between postsurgical patients with cyanotic ACHD and those with acyanotic ACHD; lipid levels increased in only $11 \%$ of the cyanotic patients who had hypocholesterolemia before surgery. The present study did not consider the pre- and postsurgical characteristics of the cyanotic patients in the analyses. Thus, it is advisable to differentiate between acyanotic and cyanotic surgically corrected ACHD patients in follow-up studies. Third, Steinberger et al. ${ }^{19)}$ stated that the atherosclerotic process begins in childhood and progresses slowly into adulthood, and that lipid levels in childhood are linked to lipid levels and obesity in adulthood. ${ }^{211}$ However, the present study measured the lipid levels and obesity at only one time point during adulthood. Therefore, it is advisable to conduct a longitudinal study of risk factors including lipid levels and obesity from childhood to adulthood.

\section{Conclusion}

On comparing the $\mathrm{CV}$ risk factors between the surgically corrected $A C H D$, cyanotic $A C H D$, and control groups, the patients in the 
surgically corrected ACHD group demonstrated lower exercise levels, higher lipid levels, and increased obesity compared with the controls and they showed higher risk of hypertension, diabetes, and hyperlipidemia. On the other hand, the patients in the cyanotic ACHD group showed lower risk of all lipid levels, hypertension, diabetes, hyperlipidemia, and metabolic disorder than the controls. Therefore, for patients with surgically corrected ACHD, there is a need to develop intervention programs and guidelines for managing risk factors, including obesity and lipid levels.

\section{Acknowledgments}

This study was supported by a grant (2010) from the Korean Society of Cardiology (No. PHO 01105711).

\section{References}

1. Lopez AD, Mathers CD, Ezzati M, Jamison DT, Murray CJ. Global and regional burden of disease and risk factors, 2001: systematic analysis of population health data. Lancet 2006;367:1747-57.

2. de Fatima Marinho de Souza M, Gawryszewski VP, Orduñez P, Sanhueza A, Espinal MA. Cardiovascular disease mortality in the Americas: current trends and disparities. Heart 2012;98:1207-12.

3. Statistics Korea. 2012 Statistics for cause of death [Internet]. [Accessed on Mar 12, 2014]. Available from: http://kostat.go.kr/portal/ korea/kor_nw/2/6/2/index.board?bmode=readctaSeq $=308559$.

4. Korean Heart Foundation. 2012 Statistics for cardiac surgery in congenital heart disease [Internet]. [Accessed on Mar 12, 2014]. Available from: http://www.heart.or.kr/korean/board/board.php?sa=listctbid=2.

5. Somerville J. Management of adults with congenital heart disease: an increasing problem. Annu Rev Med 1997;48:283-93.

6. Moon JR, Lee HJ. Transitioning adolescents with congenital heart disease in to adult health care. J Korean Pediatr Cardiol Soc 2004;8:135-41.

7. Goff DC Jr., Lloyd-Jones DM, Bennett G, et al. 2013 ACC/AHA guideline on the assessment of cardiovascular risk: a report of the American College of Cardiology/American Heart Association task force on practice guidelines. Circulation 2014;129(25 Suppl 2):S49-73.

8. Jang SY, Ju EY, Cho SI, Lee SW, Kim DK. Comparison of cardiovascular risk factors for peripheral artery disease and coronary artery disease in the korean population. Korean Circ J 2013;43:316-28.

9. World Health Organization Western Pacific Region, International Association for the Study of Obesity, International Obesity Task Force. The Asia-Pacific perspective: redefining obesity and its treatment. Sydney: Health Communications; 2000.

10. Yoon YS, Oh SW, Baik HW, Park HS, Kim WY. Alcohol consumption and the metabolic syndrome in Korean adults: the 1998 Korean National Health and Nutrition Examination Survey. Am J Clin Nutr 2004;80:217-24.

11. Babor TF, Higgins-Biddle JC, Saunders JB, Monteiro MG. The alcohol use disorders identification test - guidelines for use in primary care. Geneva: World Health Organization; 2001.
12. World Health Organization. 2010 Global recommendations on physical activity for health [Internet]. [Accessed on Mar 12, 2014]. Available from: http://whqlibdoc.who.int/publications/2010/9789241599979_ eng.pdf.

13. Expert Panel on Detection, Evaluation, and Treatment of High Blood Cholesterol in Adults. Executive summary of the third report of the National Cholesterol Education Program (NCEP) expert panel on detection, evaluation, and treatment of high blood cholesterol in adults (Adult Treatment Panel III). JAMA 2001;285:2486-97.

14. Third report of the National Cholesterol Education Program (NCEP) expert panel on detection, evaluation, and treatment of high blood cholesterol in adults (Adult Treatment Panel III). The guidelines [Internet]. [Accessed on Mar 12, 2014]. Available from: http://www.nhlbi. nih.gov/guidelines/cholesterol/index.htm.

15. Després JP, Lemieux I. Abdominal obesity and metabolic syndrome. Nature 2006;444:881-7.

16. Grundy SM. What is the contribution of obesity to the metabolic syndrome? Endocrinol Metab Clin North Am 2004;33:267-82.

17. Kim MH, Kim MK, Choi BY, Shin YJ. Prevalence of the metabolic syndrome and its association with cardiovascular diseases in Korea. J Korean Med Sci 2004;19:195-201.

18. Rhee MY, Kim JH, Kim YS, et al. High sodium intake in women with metabolic syndrome. Korean Circ J 2014;44:30-6.

19. Steinberger J, Daniels SR; American Heart Association Atherosclerosis, Hypertension, and Obesity in the Young Committee (Council on Cardiovascular Disease in the Young); American Heart Association Diabetes Committee (Council on Nutrition, Physical Activity, and Metabolism). Obesity, insulin resistance, diabetes, and cardiovascular risk in children: an American Heart Association scientific statement from the atherosclerosis, hypertension, and obesity in the young committee (Council on Cardiovascular Disease in the Young) and the diabetes committee (Council on Nutrition, Physical Activity, and Metabolism). Circulation 2003;107:1448-53.

20. Martínez-Quintana E, Rodríguez-González F, Nieto-Lago V, Nóvoa FJ, López-Rios L, Riaño-Ruiz M. Serum glucose and lipid levels in adult congenital heart disease patients. Metabolism 2010;59:1642-8.

21. Gidding SS, Bao W, Srinivasan SR, Berenson GS. Effects of secular trends in obesity on coronary risk factors in children: the Bogalusa Heart Study. J Pediatr 1995;127:868-74.

22. King VL, Hatch NW, Chan HW, de Beer MC, de Beer FC, Tannock LR. A murine model of obesity with accelerated atherosclerosis. Obesity (Silver Spring) 2010;18:35-41.

23. Vasconcelos IQ, Stabelini Neto A, Mascarenhas LP, et al. Cardiovascular risk factors in adolescents with different levels of energy expenditure. Arq Bras Cardio/ 2008;91:207-12, 227-33.

24. Varady KA, Jones PJ. Combination diet and exercise interventions for the treatment of dyslipidemia: an effective preliminary strategy to lower cholesterol levels? J Nutr 2005;135:1829-35.

25. Reybrouck T, Mertens L. Physical performance and physical activity in grown-up congenital heart disease. Eur J Cardiovasc Prev Rehabil 2005;12:498-502.

26. Fyfe A, Perloff JK, Niwa K, Child JS, Miner PD. Cyanotic congenital heart disease and coronary artery atherogenesis. Am J Cardiol 
2005;96:283-90.

27. Hait G, Corpus M, Lamarre FR, Yuan SH, Kypson J, Cheng G. Alteration of glucose and insulin metabolism in congenital heart disease. Circulation 1972:46:333-46.

28. Lundell KH, Sabel KG, Eriksson BO, Mellgren G. Glucose metabolism and insulin secretion in children with cyanotic congenital heart disease. Acta Paediatr 1997;86:1082-4.

29. Kavey RE, Allada V, Daniels SR, et al. Cardiovascular risk reduction in high-risk pediatric patients: a scientific statement from the American Heart Association Expert Panel on population and prevention science; the Councils on Cardiovascular Disease in the Young, epidemiology and prevention, nutrition, physical activity and metabolism, high blood pressure research, cardiovascular nursing, and the kidney in heart eisease; and the interdisciplinary working group on quality of care and outcomes research: endorsed by the American Academy of Pediatrics. Circulation 2006;114:2710-38. 